\title{
Inhibition of cell growth in human glioblastoma cell lines by farnesyltransferase inhibitor SCH66336
}

\author{
Tricia L. Glass, Ta-Jen Liu, and W.K. Alfred Yung ${ }^{2}$ \\ The University of Texas M.D. Anderson Cancer Center, Department of Neuro-Oncology, Houston, TX 77030
}

Ras activation occurs through stimulation of an upstream growth factor receptor such as epidermal growth factor receptor (EGFR). The ultimate effect of Ras is to induce nuclear transcription via a signaling pathway sequentially involving Raf, MAP kinase kinase (MEK), and mitogenactivated protein kinase (MAPK). To transform cells, Ras oncoproteins must be posttranslationally modified with a farnesyl group in a reaction catalyzed by farnesyl protein transferase. Farnesyltransferase inhibitors, therefore, have been proposed as potent anticancer agents. This study demonstrates the growth-inhibitory effects of farnesyltransferase inhibitor SCH66336 on human glioblastoma cell lines U-251 MG, U-251/E4 MG (a stably transfected cell line with elevated EGFR expression), and U-87 MG. As determined by (3-(4,5-dimethylthiazol-2-yl)-5-(3-carboxymethoxyphenyl-2-(4-sulfophenyl)-2H-tetraz olium, inner salt) (MTS) and viability assays, the concentration required to achieve $50 \%$ inhibition $\left(\mathrm{IC}_{50}\right)$ ranged from 30 $\mu \mathrm{M}$ (single 24 -h treatment) to $10 \mu \mathrm{M}$ (5-day treatment). U-251/E4 MG with overexpression of EGFR were more sensitive than U-251 MG parental cells. These observations were also supported by soft agar analysis. Cells treated with SCH66336 underwent G2 arrest. Western blot

Received 16 March 2000, accepted 10 April 2000.

${ }^{1}$ This work was supported in part by National Institutes of Health Grants CA-51148, CA-55261, and CA-16672 and a grant from the Gilliland Foundation.

${ }^{2}$ Address correspondence and reprint requests to W.K. Alfred Yung, Chairman Ad Interim, Department of Neuro-Oncology, Box 100, The University of Texas M.D. Anderson Cancer Center, 1515 Holcombe Blvd., Houston, TX, 77030.

${ }^{3}$ Abbreviations used are as follows: DMEM, Dulbecco's modified Eagle's medium; EGFR, epidermal growth factor receptor; FTI, farnesyltransferase inhibitor; $I C_{50}$, concentration required to achieve $50 \%$ inhibition; MAPK, mitogen-activated protein kinase; MTS, (3-(4,5-dimethylthiazol-2-yl)-5-(3-carboxymethoxyphenyl)-2-(4sulfophenyl)-2H-tetrazolium, inner salt). analysis revealed a decrease in phospho-MAPK levels upon treatment with $10 \mu \mathrm{M}$ SCH66336, whereas MAPK levels were unaffected by the drug. Interestingly, increased expression of EGFR was observed in U-251 MG and U-251/E4 MG but not in U-87 MG in the presence of the inhibitor. These results demonstrate that $\mathrm{SCH} 66336$ inhibits viability and anchorage-independent growth in a time- and dosedependent manner in glioblastoma cell lines U-251 MG, U-251/E4 MG, and U-87 MG via a signal transduction pathway involving the down-regulation of phosphoMAPK. Overexpression of EGFR appears to alter cellular sensitivity to farnesyltransferase inhibitors. This may have a particularly important implication in glioblastoma, where over $50 \%$ of tumors have amplification and overexpression of EGFR. Neuro-Oncology 2, 151-158, 2000 (Posted to Neuro-Oncology [serial online], Doc. 00-020, June 1, 2000. URL <neuro-oncology.mc.duke.edu>)

$\mathrm{R}$ as contributes to as many as $20 \%$ of all cancers and as many as $50 \%$ of the more common cancers, such as colon cancer (Bos, 1990). In many of these cancers, $R A S$ is mutated. The role of Ras in cancer is not limited to its mutational status, however, as it has been found to be overactivated as well in various types of cancer, including astrocytomas and glioblastomas (Bredel et al., 1999; Gerosa et al., 1989; Gutmann et al., 1996; Orian et al., 1992; Pollack et al., 1998). This overactivation may be caused by the deregulation of upstream proteins including growth factors and growth factor receptors such as the EGFR ${ }^{3}$ (Prigent et al., 1996; Westermark et al., 1995). Several studies conducted in recent years have shown that EGFR is overexpressed in $50 \%$ of glioblastomas (Feldkamp et al., 1999; Libermann et al., 1985; Olson et al., 1995; Steck et al., 1988; Wong et al., 1987). Furthermore, it has been shown in human malignant astrocytomas that proliferative signals from EGFR lead to activation of the Ras mitogenic pathway and ultimately proliferation of these cells (Guha et al., 1997). 
FTIs, which have become a major focus of therapeutics in recent years, were originally designed to inhibit the actions of oncogenic RAS. Binding of a growth factor such as EGF to its receptor (EGFR) triggers a series of events leading to the activation of Ras protein. When EGF binds to EGFR, a tyrosine kinase receptor, the receptor is activated through autophosphorylation, and growth factor receptor-bound protein-2 (Grb2) and son of sevenless (Sos) protein are recruited to the activated receptor to form a complex. This complex catalyzes the exchange of GDP for GTP on Ras, which activates Ras. Ras recruits Raf, a serine/threonine kinase, which is then activated and functions to phosphorylate MAP kinase kinase (MEK). MAP kinase kinase, in turn, phosphorylates MAPK on threonine and tyrosine residues. Once activated, MAPK translocates to the nucleus to phosphorylate various transcription factors and thus induces cellular proliferation (Lewin, 1997).

In order to transform cells, Ras oncoproteins must first be modified. Ras is initially formed as an inactive precursor protein in the cytoplasm of cells. A crucial step in the activation of Ras involves the transfer of a farnesyl group from farnesyldiphosphate by the enzyme farnesyltransferase. The addition of the farnesyl group occurs on the carboxyl cysteine of a CAAX sequence, where $\mathrm{C}$ is a cysteine, $\mathrm{A}$ is an aliphatic amino acid, and $\mathrm{X}$ is any amino acid except leucine or isoleucine. This farnesyl group acts as a molecular hook that allows Ras to attach to the membrane. Without this farnesyl group, Ras is biologically inactive (Travis, 1993). FTIs were designed to target the enzyme farnesyltransferase and thus block the attachment of Ras to the membrane, rendering Ras biologically inactive.

FTIs have been shown to play an important role in the inhibition of oncogenic RAS. Studies have shown that cells transformed with oncogenic RAS morphologically resemble normal, untransformed cells in the presence of the FTI. Furthermore, Ha-ras-transformed Rat1 fibroblasts were able to revert to the normal phenotype and growth pattern when treated with inhibitors of farnesyltransferase (James et al., 1993). It has recently been noted that one mechanism by which FTIs exert their effect on cellular morphology is via the extension of microtubules in the cytoplasm of cells (Suzuki et al., 1998). It has been postulated that FTIs may exert their growth-inhibitory effect via apoptosis, as these inhibitors have been shown to induce apoptosis in v-Haras-transformed Rat1 fibroblasts that have been denied substratum attachment (Lebowitz et al., 1997).

FTIs play an important role in human cancer cell lines. It has been demonstrated that FTIs are able to block anchorage-dependent and anchorage-independent growth in various human tumor cell lines, including breast, colon, prostate, pancreas, ovarian, and lung (Nagasu et al., 1995; Sepp-Lorenzino et al., 1995). Furthermore, studies have shown that FTIs are effective not only against tumors with mutant $R A S$ but also against tumors with wild-type RAS (Sepp-Lorenzino et al., 1995). In vivo, FTIs have been found to inhibit the growth of various types of $R A S$-dependent murine and human tumors in animal models with no evidence of systemic toxicity (Kohl et al., 1994, 1995; Sun et al., 1995).

Although the exact mechanism by which FTIs work is not known, investigators have shown that FTIs have limited or no effect on normal cells in vitro and in vivo (Kohl et al., 1994, 1995). This is important because cells must maintain some level of Ras activity in order to function normally. Furthermore, many other key proteins must also be farnesylated to achieve biological functionality. With these findings, FTIs have become very promising agents for the treatment of many types of cancers, including glioblastoma.

In this study, we examined the effects of SCH66336, a specific inhibitor of the protein farnesyltransferase, on human glioblastoma cell lines U-251 MG, U251/E4 MG (a stably transfected cell line with elevated EGFR expression), and U-87 MG. Here we report the anchorage-dependent and anchorage-independent growth inhibition by SCH66336 on glioblastoma cell lines U-251 MG, U251/E4 MG, and U-87 MG. The effects of SCH66336 on cell cycle phase distribution were also examined, demonstrating the inhibitor's ability to arrest cells in $G_{2}$. We also elucidated the effect of SCH66336 on EGFR, phospho-MAPK, and MAPK activities.

\section{Materials and Methods}

\section{Cell culture}

Human glioblastoma cell lines U-251 MG and U-87 MG were obtained from the American Type Culture Collection (Manassas, Va.). The U251/E4 MG cell line was produced from parental U-251 MG as a stably transfected cell line with elevated EGFR expression. All cell lines were maintained in a 1:1 mixture of Dulbecco's modified Eagle's medium (DMEM) with Ham's F-12 supplemented with high glucose and $10 \%$ fetal calf serum (Life Technologies, Grand Island, N.Y.) in a humidified atmosphere containing $5 \% \mathrm{CO}_{2}$ at $37^{\circ} \mathrm{C}$.

\section{SCH66336}

The farnesyltransferase inhibitor SCH66336 was synthesized by Schering-Plough Research Institute (Kenilworth, N.J.). A stock solution of $10 \mathrm{mM}$ was reconstituted in dimethylsulfoxide and stored at $-20^{\circ} \mathrm{C}$. Dilutions were then prepared in DMEM/F-12 (1:1)/high-glucose medium supplemented with $10 \%$ fetal calf serum (Life Technologies). Because dimethylsulfoxide at higher concentrations is known to have a toxic effect on cells, preliminary studies were conducted to rule out the effect of dimethylsulfoxide on the cell lines examined in our experiment (data not shown). Cell lines U-251 MG, U251/E4 MG, and U-87 MG were treated with various concentrations of dimethylsulfoxide ranging from $0 \%$ to $0.6 \%$. No effect on cell growth was seen in this range of concentrations. Because our studies use SCH66336 that is reconstituted in $0.05 \%$ to $0.1 \%$ dimethylsulfoxide, it was safe to conclude that any effect observed on the cells was due strictly to SCH66336.

\section{Growth Inbibition Assay-MTS Assay}

On the day before treatment began (referred to as day 0 ), cells were trypsinized and seeded in 3 triplicate sets of 96well microtiter plates at $1 \times 10^{3}$ cells/well. On day 1 , the medium was replaced with fresh medium containing SCH66336 at concentrations of $0,5,10,25$, or $50 \mu \mathrm{M}$. 
Control cells received vehicle alone. In the first set of plates, the cells were treated with fresh medium on day 2 and allowed to grow untreated until day 11 . In the second set of plates, the cells were treated daily on days 2 to 5 with the aforementioned concentrations of SCH66336. Fresh medium containing the drug was added with each daily drug application. After 5 days of treatment, fresh, inhibitor-free medium was added on day 6. Cells were allowed to grow untreated until day 11. Cells in the third set of plates were treated daily on days 2 to 10 in the same manner as the second set of plates. For all 3 sets, the number of viable cells in proliferation was determined on day 11 using a colorimetric cell proliferation assay (CellTiter 96 AQ $_{\text {ueous }}$ Non-Radioactive Cell Proliferation Assay; Promega Corp., Madison, Wis.).

\section{Cell Viability Assay}

On day 0 , cells were trypsinized and plated at $1 \times 10^{4}$ cells/well in triplicate in 6-well plates. On day 1 , the medium was replaced with fresh medium containing $5 \mu \mathrm{M}$ or $10 \mu \mathrm{M}$ SCH66336. Control cells received vehicle alone. Daily treatment with the inhibitor continued on days 2 to 5. Cells were given fresh medium with each application of the drug. Viable cells were scored manually on day 6 .

\section{Anchorage-Independent Growth Assay}

Cells were seeded at $1 \times 10^{4}$ cells/well in 6 -well plates on day 0 . On day 1 , the medium was replaced with fresh medium containing 0,5 , or $10 \mu \mathrm{M}$ SCH66336. Cells were treated with the inhibitor daily on days 2 to 5 . Fresh medium was added with each application of the drug. On day 6 , cells were washed with inhibitor-free medium and trypsinized, and $1 \times 10^{3}$ cells/well were seeded in 6-well plates in $0.65 \%$ agar over a $0.9 \%$ agar layer. After 2 weeks of growth, $1.5 \mathrm{ml}$ medium was added to each well. Colonies were scored manually after 4 weeks of growth. All studies were conducted in triplicate.

\section{Flow Cytometric Analysis}

On day 0 , cells were seeded in $100-\mathrm{mm}$ plates at 1 to $2 \times$ $10^{5}$ cells/plate in 2 triplicate sets. Cells were treated daily with fresh medium containing 5 or $10 \mu \mathrm{M}$ SCH66336 for 5 days beginning on day 1 and continuing through day 5 . Control cells received vehicle alone. On day 6, 1 set of plates was harvested. The other set of plates was given fresh inhibitor-free medium and harvested on day 8. Upon collection, cells were washed twice with phosphatebuffered saline and fixed in $70 \%$ ethanol overnight. After fixation, the cells were washed twice with phosphatebuffered saline. The cells were then stained in phosphatebuffered saline containing $50 \mu \mathrm{g} / \mathrm{ml}$ propidium iodide and $20 \mu \mathrm{g} / \mathrm{ml}$ RNAse for $15 \mathrm{~min}$ at room temperature. Fluorescence-activated cell sorting was done using an EPICS profile flow cytometer (Coulter Corp., Hialeah, Fla.)

\section{Western Blot Analysis}

Cell lines U-251 MG, U251/E4 MG, and U-87 MG were seeded in 6 -well plates on day 0 at $5 \times 10^{4}$ cells/well. All cell lines were treated daily with fresh medium containing $10 \mu \mathrm{M}$ SCH66336 on days 1 to 5 . Control cells received vehicle alone. On day 6 , cells were washed 3 times with $2 \mathrm{ml} \mathrm{DMEM} / \mathrm{F}-12$ (1:1)/high glucose. After washing, $2 \mathrm{ml}$ DMEM/F-12 (1:1)/high glucose was added to each well. Cells were incubated at $37^{\circ} \mathrm{C}$ for $6 \mathrm{~h}$. After the 6-h incubation, the medium was removed and replaced with $1 \mathrm{ml}$ DMEM/F-12 (1:1)/high glucose/2\% fetal calf serum (Life Technologies). Cultures were incubated at $37^{\circ} \mathrm{C}$ for $24 \mathrm{~h}$. After $24 \mathrm{~h}$, cells were collected and washed in phosphate-buffered saline. Cells were lysed in RIPA buffer $(150 \mathrm{mM} \mathrm{NaCl} ; 1 \% \mathrm{NP}-40 ; 0.5 \%$ DOC; $0.1 \%$ sodium dodecyl sulfate; $50 \mathrm{mM}$ Tris, $\mathrm{pH}$ 8.0) containing protease inhibitors (protease inhibitor set; Boehringer Mannheim, Indianapolis, Ind.). Cell lysates were clarified by centrifugation and quantitated by Bradford assay. Cellular protein $(30 \mu \mathrm{g})$ was heated at $98^{\circ} \mathrm{C}$, immediately fractionated on a $10 \%$ Tris- $\mathrm{HCl}$ polyacrylamide gel (Bio-Rad, Richmond, Calif.), and then transferred to Hybond ECL nitrocellulose membrane (Amersham Life Science, Arlington Heights, Ill.). The p44/42 anti-MAPK rabbit polyclonal immunoglobulin-G antibody and the phospho-specific p44/42 MAPK rabbit polyclonal immunoglobulin-G antibody (New England Biolabs, Beverly, Mass.) were used as recommended by the vendor, with a 1:2000 dilution of antirabbit immunoglobulin-G horseradish peroxidase-conjugated secondary antibody (Amersham Life Science). The antiEGFR human (Ab-4, rabbit) antibody (Calbiochem, San Diego, Calif.) was used per manufacturer's instructions in conjunction with a 1:1000 dilution of the aforementioned rabbit secondary antibody. Protein bands were visualized using a commercially available ECL kit (Amersham Life Science), and densitometry was performed using an Alpha Imager 2000 Documentation and Analysis System (Alpha Innotech Corp., San Leandro, Calif.).

\section{Results}

\section{SCH66336 Inhibits Cell Growth-MTS Assay}

To examine the effect of SCH66336 on cell growth, U-251 MG, U251/E4 MG, and U-87 MG cells were treated with $0,1,5,10,25$, or $50 \mu \mathrm{M} \mathrm{SCH} 66336$ for 1 , 5 , or 10 days (Fig. 1). Our initial studies using the MTS assay found that exposure to $\mathrm{SCH} 66336$ produced a dramatic dose- and time-dependent decrease in cellular proliferation. After treating U-87 MG cells for $24 \mathrm{~h}$ with this compound, growth of U-87 MG was inhibited by $36 \%$ in $10 \mu \mathrm{M}$ SCH66336 and by $42 \%$ in $25 \mu \mathrm{M}$ SCH66336 (Fig. 1C). Growth of U-87 MG cells was inhibited by $43 \%$ and $94 \%$ after 5 days in 10 or $25 \mu \mathrm{M} \mathrm{SCH} 66336$, respectively (Fig. 1C). Similar trends were observed in U$251 \mathrm{MG}$ and U251/E4 MG (Fig. $1 \mathrm{~A}$ and $\mathrm{B}$ ). $\mathrm{IC}_{50}$ ranged from $30 \mu \mathrm{M}$ (single 24 -h treatment) to $10 \mu \mathrm{M}$ (5-day treatment). Sensitivity varied among the cell lines, with U-87 MG exhibiting the most sensitivity to the drug, and U251/E4 MG cells exhibiting more sensitivity than the U$251 \mathrm{MG}$ parental cells (Fig. 1). 

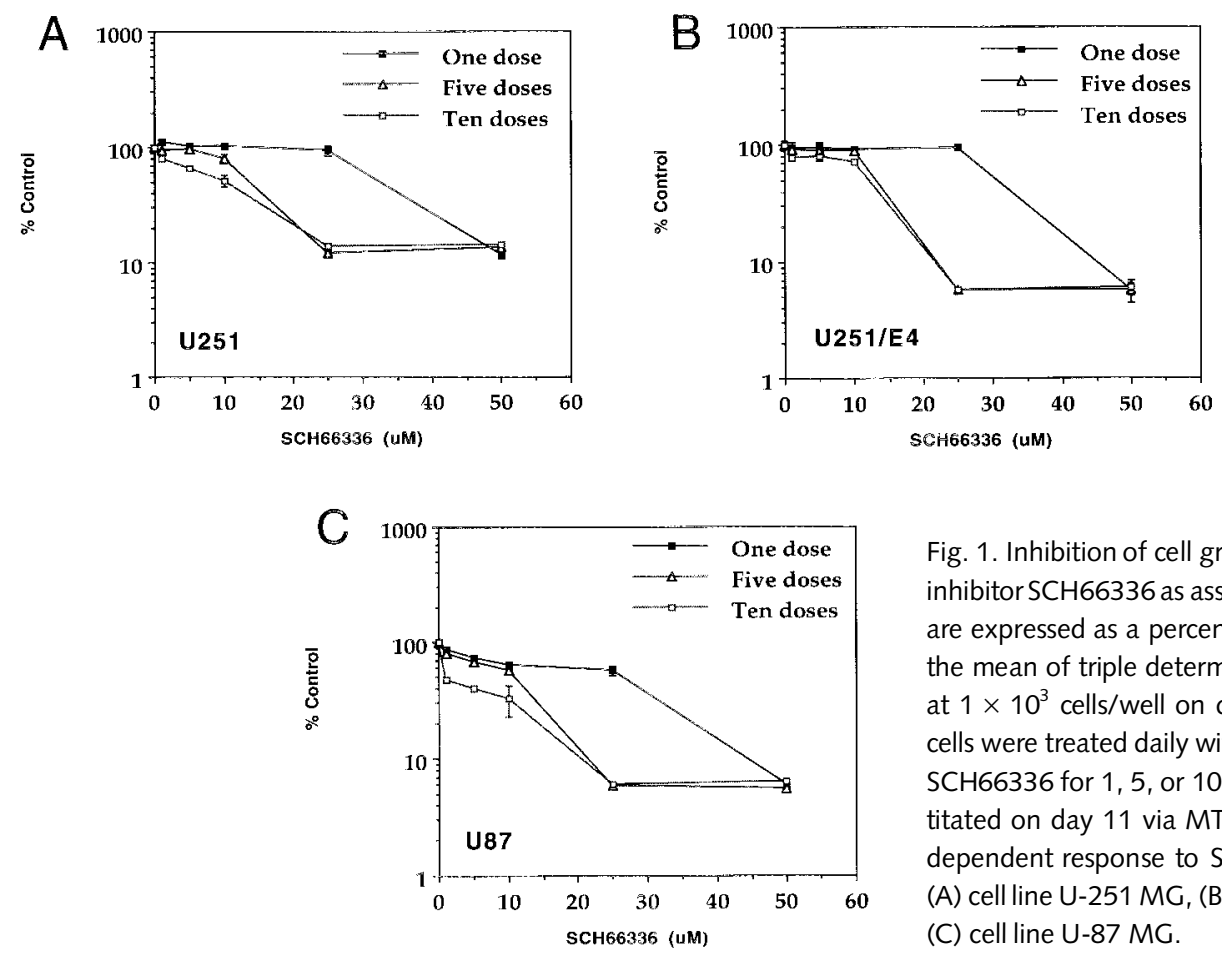

Fig. 1. Inhibition of cell growth by farnesyltransferase inhibitor SCH66336 as assessed by MTS assay. Results are expressed as a percentage of the control and are the mean of triple determinations. Cells were seeded at $1 \times 10^{3}$ cells/well on day 0 . Beginning on day 1 , cells were treated daily with $0,1,5,10,25$, or $50 \mu \mathrm{M}$ $\mathrm{SCH} 66336$ for 1,5 , or 10 days. All plates were quantitated on day 11 via MTS assay. A time- and dosedependent response to SCH66336 was exhibited in (A) cell line U-251 MG, (B) cell line U251/E4 MG, and (C) cell line U-87 MG.

\section{SCH66336 Inhibits Cell Viability}

To determine whether anchorage-dependent growth was inhibited by the FTI, cells were treated daily with fresh medium containing 5 or $10 \mu \mathrm{M} \mathrm{SCH} 66336$ for 5 days (Fig. 2). Cell viability was inhibited by $70 \%$ and $76 \%$ in U-251 MG when treated with 5 or $10 \mu \mathrm{M}$ SCH66336, respectively. Similar trends were observed in U251/E4 MG cells, which were inhibited by $65 \%$ and $78 \%$ when treated with $5 \mu \mathrm{M}$ or $10 \mu \mathrm{M}$ SCH66336, respectively. The inhibitor's effect was most pronounced in the U-87 MG cell line, which showed $93 \%$ growth inhibition when treated with $5 \mu \mathrm{M} \mathrm{SCH} 66336$ and $95 \%$ growth inhibition when treated with $10 \mu \mathrm{M}$ SCH66336. Again, all cell lines demonstrated dose-dependent growth inhibition.

\section{SCH66336 Inhibits Anchorage-Independent Cell Growth}

We tested the effect SCH66336 had on the ability of human tumor cell lines to form colonies in soft agar (Fig. 3). Cells were treated with 5 or $10 \mu \mathrm{M}$ SCH66336 in fresh medium daily for 5 days. Control cells received vehicle alone. On day 6 , cells were seeded in soft agar. Clonogenicity in U-87 MG cells was inhibited by $64 \%$ and $67 \%$ in 5 or $10 \mu \mathrm{M}$ SCH66336, respectively. A similar effect was seen in U251/E4 MG, which was inhibited by $67 \%$ and $75 \%$ in 5 or $10 \mu \mathrm{M}$ SCH66336, respectively. Alternatively, cell line U$251 \mathrm{MG}$ was more resistant than the others, with 5 and 10 $\mu \mathrm{M}$ SCH66336 inhibiting clonogenicity by $24 \%$ and $45 \%$.

\section{SCH66336 Stimulates $G_{2}$ Arrest in Glioblastoma Cell Lines}

To test the effect of SCH66336 on cell cycling, cells were treated with $5 \mu \mathrm{M}$ or $10 \mu \mathrm{M} \mathrm{SCH} 66336$ in fresh medium daily for 5 days. Cells were harvested on days 6 and 8 , stained with propidium iodide, and analyzed by fluorescence-activated cell sorting (Fig. 4). Addition of $10 \mu \mathrm{M}$ SCH66336, a dose shown to have a pronounced effect on cell growth, had no significant effect on the percentage of cells in the sub $G_{0} / G_{1}$ phase of the cell cycle. However, cells treated with 5 and $10 \mu \mathrm{M}$ of the drug showed a marked decrease in the number of cells in $G_{1}$. This decrease in $G_{1}$ cells was seen in cells that were harvested at both time points. A subsequent increase in the number of cells in the $G_{2}$ phase was seen in all 3 cell lines treated with both con-

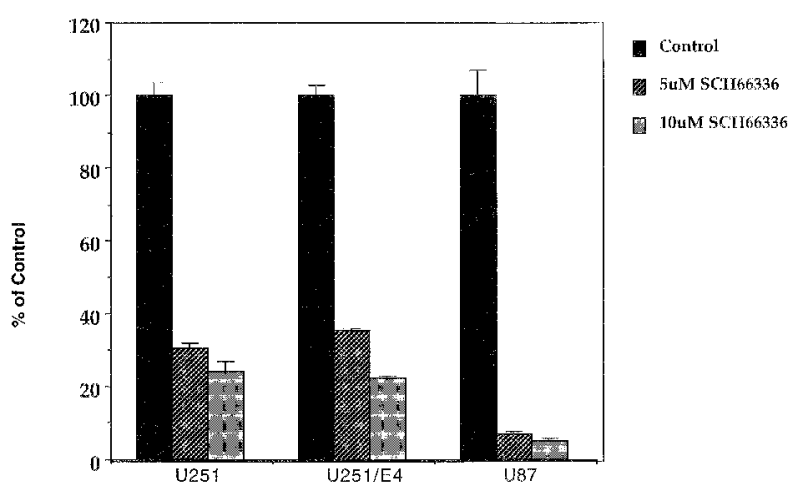

Fig. 2. SCH66336 inhibition of cell viability in glioblastoma cell lines U251 MG, U251/E4 MG, and U-87 MG. Cells were seeded on day 0 at $1 \times 10^{4}$ cells/well. Beginning on day 1 , cells were treated with 5 or $10 \mu \mathrm{M} \mathrm{SCH} 66336$ daily for 5 days and counted on day 6 . Control cells were treated with vehicle alone. Results are expressed as a percentage of the control $( \pm S D)$ and are the mean of triplicate determinations. Response of the cell lines to the drug occurred in a time- and dosedependent fashion. 


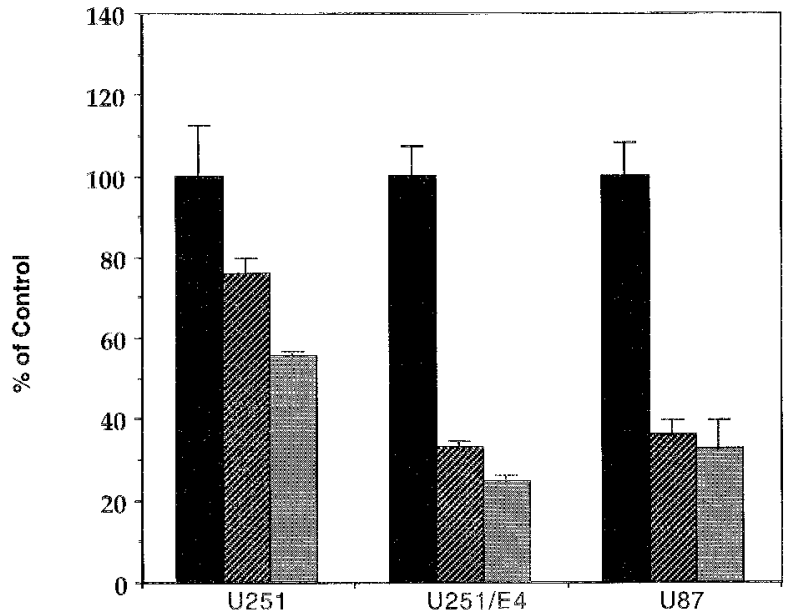

- OuM SCH66336

5uM SCH 66336

图 10uM SCH66336

Fig. 3. Anchorage-independent growth inhibition by SCH66336. On day 0, cells were seeded at $1 \times 10^{4}$ cells/well. Cells were treated with 5 or $10 \mu \mathrm{M} \mathrm{SCH} 66336$ daily for 5 days beginning on day 1 . Fresh medium was added with each application of the drug. Control cells received vehicle alone. On day 6 , cells were trypsinized, and $1 \times 10^{3}$ cells/well were seeded in $0.65 \%$ agar over a $0.9 \%$ agar layer. Colonies were scored manually after 4 weeks of growth. Results are expressed as a percentage of the control $( \pm S D)$ and are the mean of triplicate determinations. Anchorage-independent growth was inhibited in all cell lines upon addition of SCH66336 with the least inhibition occurring in U-251 MG cells. centrations of the inhibitor. The increase in $G_{2}$ was dose dependent, with U-87 MG exhibiting the most dramatic response to the FTI. In this cell line, the percentage of cells harvested on day 6 in $\mathrm{G}_{2}$ treated with 5 or $10 \mu \mathrm{M}$ SCH66336, increased by $45 \%$ and $51 \%$, respectively. U87 MG cells harvested on day 8 and treated with 5 and 10 $\mu \mathrm{M}$ of the inhibitor showed an increase of $35 \%$ and $43 \%$, respectively, in the number of cells in $\mathrm{G}_{2}$. A slight increase in the number of cells in $S$ phase, when treated with either concentration of the FTI, was seen in U-251 MG and U251/E4 MG, but not in U-87 MG.

\section{SCH66336 Reduces Phospho-MAPK Activity but Not MAPK Activity}

To examine the effect of SCH66336 on MAPK and phospho-MAPK expression, cells were treated with $10 \mu \mathrm{M}$ SCH66336 daily for 5 days. Fresh medium was added
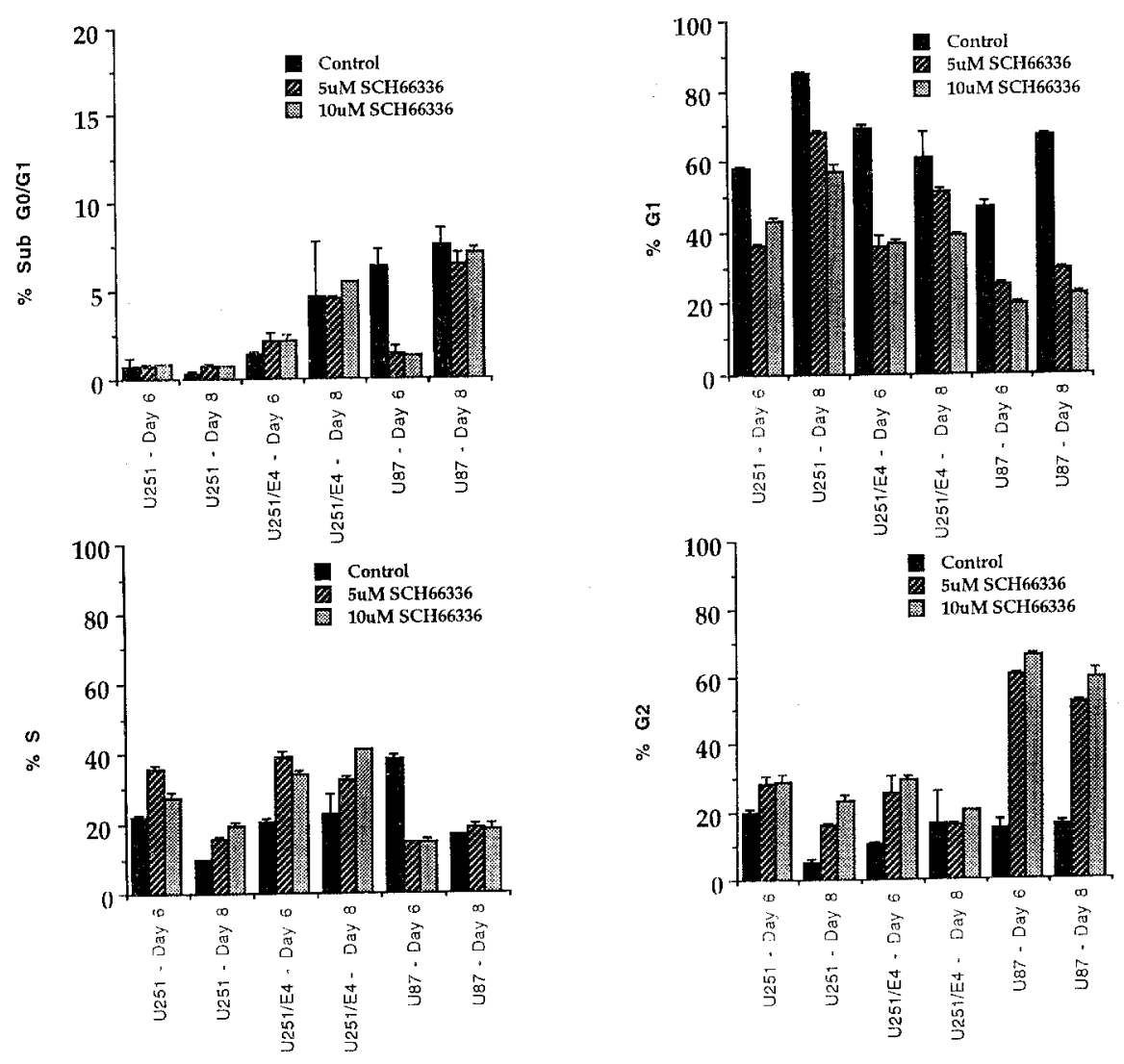

Fig. 4. $\mathrm{G}_{2}$ arrest of cells undergoing SCH66336 treatment. On day 0 , cells were seeded at 1 to $2 \times 10^{5}$ cells/plate in 2 triplicate sets. Cells were treated with fresh medium containing 5 or $10 \mu \mathrm{M} \mathrm{SCH} 66336$ for 5 consecutive days. Control cells received vehicle alone. Cells were harvested on days 6 and 8 and analyzed by fluorescence-activated cell sorting. Cell cycle analysis revealed an induction of $\mathrm{G}_{2}$ arrest in the presence of SCH66336. Bars are \pm SD. 
with each addition of the drug. Cells were washed, preconditioned with serum-free media, and conditioned with medium containing $2 \%$ fetal calf serum on day 6 . Cells were collected on day 7 and analyzed via Western blot analysis (Fig. 5), which revealed no change in MAPK levels in the 3 cell lines in the presence of the drug. Furthermore, all of the cell lines exhibited similar levels of endogenous MAPK protein. However, a decrease in phospho-MAPK expression levels was observed in all of the cell lines upon exposure to $10 \mu \mathrm{M}$ SCH66336. Up to a 2fold decrease in the level of expression of phospho-MAPK levels was observed in cells treated with the drug. Basal phospho-MAPK levels in control cells varied among the cell lines, with U-87 MG exhibiting the lowest level of phospho-MAPK and U-251 MG exhibiting the highest. The effect of SCH66336 on EGFR protein levels was dependent on the cell line. The amount of EGFR protein in cell line U-87 MG remained unchanged in the presence of the drug. Cell lines U-251 MG and U251/E4 MG, however, exhibited an increase in EGFR levels upon treatment with the drug. Endogenous EGFR levels again varied among the cell lines, with the lowest expression level in U-87 MG and the highest in U251/E4 MG.

\section{Discussion}

The data presented here demonstrate that SCH66336 is capable of inhibiting anchorage-dependent growth in the 3 glioblastoma cell lines tested. Response of cells to the drug occurred in a time- and dose-dependent fashion. $\mathrm{IC}_{50}$, according to the MTS assay, ranged from $30 \mu \mathrm{M}$ (single 24 -h treatment) to $10 \mu \mathrm{M}$ (5-day treatment). It was determined that continuous drug exposure for several days was necessary to achieve optimum cytotoxic effects. Sensitivity among the cell lines differed, with cell line U-87 MG displaying the greatest sensitivity. Interestingly, cell line U251/E4 MG was more susceptible to the inhibitor than U-251 MG parental cells. Based on observations from the MTS assay, the optimal treatment condition appeared to be at a concentration of either 5 or 10 $\mu \mathrm{M}$ for a period of 5 days. Therefore, cells in the remaining experiments were treated in this manner prior to analysis. Viability assays confirmed the anchorage-dependent growth inhibition of SCH66336 on cell lines U-251 MG, U251/E4 MG, and U-87 MG.

Soft agar analysis showed that SCH66336 was also able to inhibit anchorage-independent growth in all 3 cell lines. Response of U251/E4 MG and U-87 MG to the drug was similar. Cell line U-251 MG was again the least sensitive to the drug. Clonogenic response of the cell lines to the drug occurred in a time- and dose-dependent fashion.

Results obtained from anchorage-dependent and -independent assays are consistent with previous experiments, which have demonstrated the ability of FTIs to block anchorage-dependent and -independent growth in various human tumor cell lines, including breast, prostate, colon, pancreas, ovarian, and lung, regardless of $R A S$ status (Nagasu et al., 1995; Sepp-Lorenzino et al., 1995). Recent studies also support the observation of anchorage-dependent growth inhibition of glioblastoma cell lines by FTIs (Bredel et al., 1998).

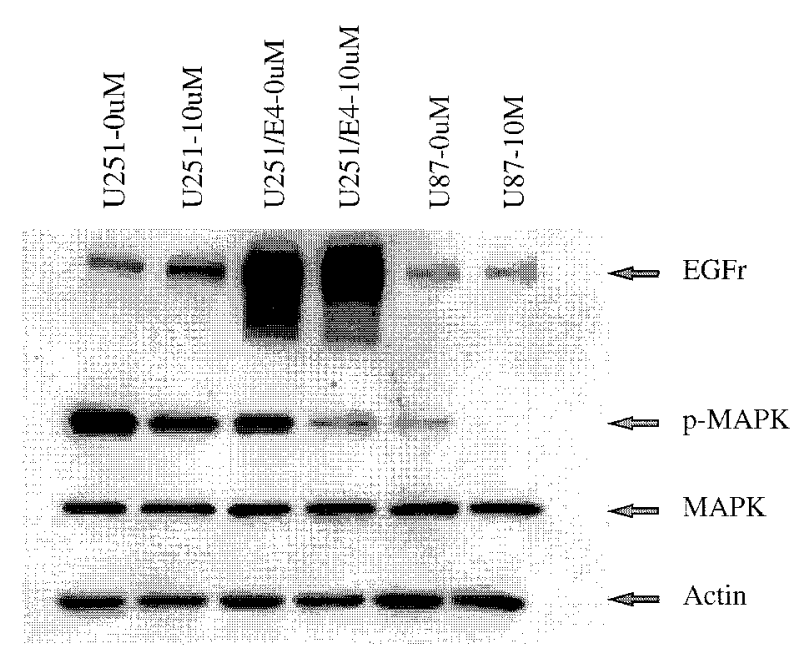

Fig. 5. Downregulation of phospho-MAPK by SCH66336. Cells were plated on day 0 at $5 \times 10^{4}$ cells/well. On days 1 to 5 , cells were treated with fresh medium containing $10 \mu \mathrm{M} \mathrm{SCH66336.} \mathrm{Cells} \mathrm{were}$ washed, preconditioned, and conditioned on day 6 and collected on day 7. Total cellular protein was analyzed via Western blot analysis. Phospho-MAPK levels decreased upon exposure to SCH66336, whereas total MAPK levels remained unchanged. In the presence of inhibitor, EGFR levels increased in cell lines U-251 MG and U251/E4, but were unaffected in cell line U-87 MG.

Flow cytometric studies showed that SCH66336 treatment induces $G_{2}$ arrest in all 3 of the glioblastoma cell lines tested. The effects of FTIs on cell cycling have been previously documented. A recent study demonstrated that in lung carcinoma and fibrosarcoma cells, FTI-277 caused an accumulation of cells in $\mathrm{G}_{2} / \mathrm{M}$ (Vogt et al., 1997). Our experiments showed that $G_{2}$ accumulation in cells treated with SCH66336 was dose-dependent. Furthermore, SCH66336 again had the most pronounced effect on cell line U-87 MG, where the percentage of cells in $\mathrm{G}_{2}$ on day 6 increased from $15 \%$ to $61 \%$ and $66 \%$ after treatment with 5 or $10 \mu \mathrm{M}$ SCH66336, respectively. In our experiment, no apoptosis was observed in cells treated with 10 $\mu \mathrm{M}$ SCH66336, a dose that had previously been shown to have a notable effect on cell growth. In literature, the ability of FTIs to induce apoptosis varies according to conditions under which they are used. For example, recent studies have shown that FTIs do not induce apoptosis of Ras-transformed cells that are in contact with the substratum, but do cause apoptosis when these same cells are denied substratum attachment (Lebowitz et al., 1997).

To investigate the mechanisms accounting for the cellular effects of the FTI, we examined the effect of the inhibitor on expression levels of 3 proteins involved in the Ras signaling pathway. In all 3 glioblastoma cell lines, SCH66336 was found to cause a decrease in endogenous phospho-MAPK expression levels (the activated form of the enzyme). Our results are consistent with the report that treatment with FTI causes a reduction of MAPK activity (Nagase et al., 1996). SCH66336 was found to cause up to a 2-fold reduction in phospho-MAPK expression levels. These observations demonstrate that inhibition of farnesyltransferase blocks Ras processing, thus preventing the activation of downstream proteins that are 
needed to phosphorylate and activate MAPK. Untreated cell line U-87 MG was found to express the lowest levels of endogenous phospho-MAPK among the cell lines. Levels of phospho-MAPK were essentially undetectable in U-87 MG cells upon treatment with $10 \mu \mathrm{M}$ SCH66336. Interestingly, this cell line has consistently been the most sensitive of the 3 cell lines. The difference in levels of a protein downstream of Ras may account for the varying degrees of resistance in the cell lines. Because of intrinsically low phospho-MAPK levels in U-87 MG, the growth inhibitory effect of the drug is even more pronounced on this cell line as compared with cells that have higher levels of endogenous phospho-MAPK. In addition, cell line U251/E4 MG, which is generally more sensitive to SCH66336 than the U-251 MG parental cells, was found to possess lower endogenous levels of phospho-MAPK than the U-251 MG cells. The same explanation for differences in sensitivity would also apply here.

Next, we examined the levels of endogenous total MAPK protein in the glioblastoma cell lines. Upon treatment with the inhibitor, MAPK levels remained unchanged. Furthermore, intrinsic MAPK levels were similar for the 3 cell lines. The MAPK antibody is phosphorylation-state independent and thus recognizes both the phosphorylated (active) and unphosphorylated (inactive) forms of MAPK. Upon treatment with SCH66336, MAPK levels remained unchanged. This can be explained by the fact that although the total level of MAPK in the cells remained the same, most of the MAPK consisted of the unphosphorylated form. This explanation is supported by results obtained from the previously mentioned phospho-MAPK analysis.
Finally, EGFR protein levels in the 3 cell lines were assessed by Western blot analysis. EGFR expression levels were highest in cell line U251/E4 MG, as expected. U-87 MG expressed the lowest levels of EGFR of the 3 cell lines. Upon treatment with $10 \mu \mathrm{M}$ SCH66336, EGFR levels increased in cell lines U-251 MG and U251/E4 MG. Previous studies have shown that EGFR is downregulated as part of a Ras-dependent negative feedback loop (Klarlund et al., 1995; Osterop et al., 1993). Thus, the increase in EGFR seen in cell lines U-251 MG and U251/E4 MG may function to compensate for the inhibition of Ras after treatment with $10 \mu \mathrm{M}$ SCH66336. Interestingly, in the presence of $10 \mu \mathrm{M}$ SCH66336, EGFR expression levels in U-87 MG remained unchanged. This phenomenon may be explained by the fact that the Ras pathway is not as active in this cell line (as previously shown here). Therefore, this negative feedback loop may not be as active and may not have the same degree of sensitivity to SCH66336 as cell lines U-251 MG and U251/E4 MG.

Throughout these experiments, we observed that U251/E4 MG cells with overexpression of EGFR are more sensitive to SCH66336 than are U-251 MG parental cells. Thus, overexpression of EGFR appears to alter cellular sensitivity to FTI. This observation may be of great clinical importance in the treatment of glioblastoma, as $40 \%$ to $50 \%$ of glioblastomas have been found to overexpress EGFR, which leads to elevated levels of Ras GTP (Feldkamp et al., 1999; Guha et al., 1997; Libermann et al., 1985; Olson et al., 1995; Steck et al., 1988; Wong et al., 1987). Therefore, FTIs offer a very feasible and promising approach in the treatment of glioblastoma. SCH66336 is presently being evaluated in phase I clinical trials.

\section{References}

Bos, J.L. (1990) Ras gene mutations and human cancer. In: Cossman, J. (Ed.), Molecular Genetics in Cancer Diagnosis. Amsterdam: Elsevier Scientific Publishing Co. pp. 273-287.

Bredel, M., and Pollack, I.F. (1999) The p21-Ras signal transduction pathway and growth regulation in human high-grade gliomas. Brain Res. Rev. 29, 232-249.

Bredel, M., Pollack, I.F., Freund, J.M., Hamilton, A.D., and Sebti, S.M. (1998) Inhibition of Ras and related G-proteins as a therapeutic strategy for blocking malignant glioma growth. Neurosurgery 43, 124-131.

Feldkamp, M.M., Lala, P., Lau, N., Roncari, L., and Guha, A. (1999) Expression of activated epidermal growth factor receptors, Ras-guanosine triphosphate, and mitogen-activated protein kinase in human glioblastoma multiforme specimens. Neurosurgery 45, 1442-1453.

Gerosa, M.A., Talarico, D., Fognani, C., Raimondi, E., Colombatti, M., Tridente, G., De Carli, L., and Della Valle, G. (1989) Overexpression of N-ras oncogene and epidermal growth factor receptor gene in human glioblastomas. J. Natl. Cancer Inst. 81, 63-67.

Guha, A., Feldkamp, M.M., Lau, N., Boss, G., and Pawson, A. (1997) Proliferation of human malignant astrocytomas is dependent on Ras activation. Oncogene 15, 2755-2765.

Gutmann, D.H., Giordano, M.J., Mahadeo, D.K., Lau, N., Silbergeld, D., and Guha, A. (1996) Increased neurofibromatosis 1 gene expression in astrocytic tumors: Positive regulation by p21 ras. Oncogene 12, 2121-2127.

James, G.L., Goldstein, J.L., Brown, M.S., Rawson, T.E., Somers, T.C., McDowell, R.S., Crowley, C.W., Lucas, B.K., Levinson, A.D., and Marsters, J.C., Jr. (1993) Benzodiazepine peptidomimetics: Potent inhibitors of Ras farnesylation in animal cells. Science 260, 1937-1942.
Klarlund, J.K., Cherniack, A.D., and Czech, M.P. (1995) Divergent mechanisms for homologous desensitization of $\mathrm{p} 21^{\text {ras }}$ by insulin and growth factors. J. Biol. Chem. 270, 23421-23428.

Kohl, N.E., Wilson, F.R., Mosser, S.D., Giuliani, E., deSolms, S.J., Conner, M.W., Anthony, N.J., Holtz, W.J., Gomez, R.P., Lee, T.J., Smith, R.L., Graham, S.L., Hartman, G., Gibbs, J.B., and Oliff, A. (1994) Protein farnesyltransferase inhibitors block the growth of Ras-dependent tumors in nude mice. Proc. Natl. Acad. Sci. U.S.A. 91, 9141-9145.

Kohl, N.E., Omer, C.A., Conner, M.W., Anthony, N.J., Davide, J.P., deSolms, S.J., Guiliani, E.A., Gomez, R.P., Graham, S.L., Hamilton, K., Handt, L.K., Hartman, G.D., Koblan, K.S., Kral, A.M., Miller, P.J., Mosser, S.O., O'Neill, T.J., Rands, E., Schaber, M.D., Gibbs, J.B., and Oliff, A. (1995) Inhibition of farnesyltransferase induces regression of mammary and salivary carcinomas in ras transgenic mice. Nat. Med. 1, 792-797.

Lebowitz, P.F., Sakamuro, D., and Prendergast, G.C. (1997) Farnesyl transferase inhibitors induce apoptosis of Ras-transformed cells denied substratum attachment. Cancer Res. 57, 708-713.

Lewin, B. (1997) Signal transduction. In: Lewin, B. (Ed.), Genes VI. New York: Oxford University Press Inc. pp. 1054-1085.

Libermann, T.A., Nusbaum, H.R., Razon, N., Kris, R., Lax, I., Soreq, H., Whittle, N., Waterfield, M.D., Ullrich, A., and Schlessinger, J. (1985) Amplification, enhanced expression, and possible rearrangement of EGF receptor gene in primary human brain tumours of glial origin. Nature $313,144-$ 147.

Nagase, T., Kawata, S., Tamura, S., Matsuda, Y., Inui, Y., Yamasaki, E., Ishiguro, H., Ito, T., and Matsuzawa, Y. (1996) Inhibition of cell growth of human hepatoma cell line (Hep G2) by a farnesyl protein transferase 
T.L. Glass et al.: SCH66336-inhibited glioblastoma cell growth

inhibitor: A preferential suppression of Ras farnesylation. Int. J. Cancer 65 , 620-626.

Nagasu, T., Yoshimatsu, K., Rowell, C., Lewis, M.D., and Garcia, A.M. (1995) Inhibition of human tumor xenograft growth by treatment with the farnesyl transferase inhibitor B956. Cancer Res. 55, 5310-5314.

Olson, J.J., James, C.D., Krisht, A., Barnett, D., and Hunter, S. (1995) Analysis of epidermal growth factor receptor gene amplification and alteration in stereotactic biopsies of brain tumors. Neurosurgery 36, 740-748.

Orian, J.M., Vasilopoulos, K., Yoshida, S., Kaye, A.H., Chow, C.W., and Gonzales, M.F. (1992) Overexpression of multiple oncogenes related to histological grade of astrocytic glioma. Br. J. Cancer 66, 106-112.

Osterop, A.P., Medema, R.H., v.d. Zon, G.C., Bos, J.L., Moller, M., and Maasen, J.A. (1993) Epidermal-growth-factor receptors generate Ras.GTP more efficiently than insulin receptors. Eur. J. Biochem. 212, 477-482.

Pollack, I.F., Bredel, M., and Erff, M. (1998) Application of signal transduction inhibition as a therapeutic strategy for central nervous system tumors. Pediatr. Neurosurg. 29, 228-244.

Prigent, S.A., Nagane, M., Lin, H., Huvar, I., Boss, G.R., Feramisco, J.R., Cavenee, W.K., and Huang H.S. (1996) Enhanced tumorigenic behavior of glioblastoma cells expressing a truncated epidermal growth factor receptor is mediated through the Ras-shc-grb2 pathway. J. Biol. Chem. 271, 25639-25645.

Sepp-Lorenzino, L., Ma, Z., Rands, E., Kohl, N.E., Gibbs, J.B., Oliff, A., and Rosen, N. (1995) A peptidomimetic inhibitor of farnesyl:protein transferase blocks the anchorage-dependent and-independent growth of human tumor cell lines. Cancer Res. 55, 5302-5309.

Steck, P.A., Lee, P., Hung, M.C., and Yung, W.K. (1988) Expression of an altered epidermal growth factor receptor by human glioblastoma cells. Cancer Res. 48, 5433-5439.

Sun, J., Qian, Y., Hamilton, A.D., and Sebti, S.M. (1995) Ras CAAX peptidomimetic FTI 276 selectively blocks tumor growth in nude mice of a human lung carcinoma with K-ras mutation and p53 deletion. Cancer Res. 55, 4243-4247.

Suzuki, N., Del Villar, K., and Tamanoi, F. (1998) Farnesyltransferase inhibitors induce dramatic morphological changes of KNRK cells that are blocked by microtubule interfering agents. Proc. Natl. Acad. Sci. U.S.A. 95, 1049910504.

Travis, J. (1993) Novel anticancer agents move closer to reality. Science $\mathbf{2 6 0}$, 1877-1878.

Vogt, A., Sun, J., Qian, Y., Hamilton, A.D., and Sebti, S.M. (1997) The geranylgeranyltransferase-l inhibitor GGTI-298 arrests human tumor cells in $\mathrm{G}_{0} / \mathrm{G}_{1}$ and induces P21 WAF1/CIP1/SD11 in a p53-dependent manner. J. Biol. Chem. 272, 27224-27229.

Westermark, B., Heldin, C.H., and Nister, M. (1995) Platelet-derived growth factor in human glioma. Glia 15, 257-263.

Wong, A.J., Bigner, S.H., Bigner, D.D., Kinzler, K.W., Hamilton, S.R., and Vogelstein, B. (1987) Increased expression of the epidermal growth factor receptor gene in malignant gliomas is invariably associated with gene amplification. Proc. Natl. Acad. Sci. U.S.A. 84, 6899-6903. 